\title{
Convergence Rates to Optimal Distribution of the Boltzmann Machine on Simulated Annealing
}

\author{
Hajime URAKAWA* \\ Division of Mathematics, Graduate School of Information Sciences, Tohoku University, \\ Aoba 6-3-09, Sendai, 980-8579, Japan
}

Received November 17, 2008; final version accepted March 2, 2009

\begin{abstract}
In this paper, explicit estimations of the convergence rates of the Boltzmann machine on simulated annealing are given. As its applications, quantitative bounds of the temperature and the transition times of the Boltzmann machine to optimal distribution are given.
\end{abstract}

KEYWORDS: Boltzmann machine, Markov chain, Gibbs matrix, total variation distance, convergence rates

\section{Introduction}

Since the nineteen eighties, many studies of simulated annealing, so called the Boltzmann machine searching the minimum of a given function have been done. But, effective estimation of the convergence rates might be so far still unknown. However, the convergence rates of Markov chains have widely been studied (cf. [2], [3], [4], [5], [7], [10], [11], [12], [13], [14], [15], [16], [18]). In this paper, applying the result of Diaconnis and Stroock [3] on the estimate of the convergence rates of Markov chains, we give an explicit estimation of the convergence rates to the optimal distribution of the Boltzmann machine (cf. Theorem 1.2). Moreover, we give concrete estimations of the temperature and transition time of the Gibbs-Boltzmann distributions of simulated annealing to optimal distribution in the simplest cases in which the number of states are four or eight (cf. Theorem 1.3 and Proposition 4.1). Even though the simplest case, our situation is completely general, and our estimation explains very well the character of the Boltzmann machine (See Remark 1.4).

Let us explain our situation, more precisely. We consider a function $F$ on a finite set $S$ having a minimum $F_{\min }$ on a subset $S_{0}$ of $S$. Let us consider an optimal distribution $q_{0}$ given by

$$
q_{0}(x):=\left\{\begin{array}{cc}
\frac{1}{\left|S_{0}\right|}, & x \in S_{0}, \\
0, & x \in S \backslash S_{0} .
\end{array}\right.
$$

It is well known (cf. see [1], for example) that

(1) the Gibbs distribution $q_{T}$ with temperature $T>0$ converges to $q_{0}$ when $T$ tends to 0 , and

(2) the distribution $r G_{T}^{m}$ converges to the Gibbs distribution $q_{T}$ when $m$ tends to infinity for every initial distribution $r$.

But, so far, it has been unknown how much $T$ and $m$ one should take rigorously in order to approximate $q_{T}$ and $r G_{T}{ }^{m}$ enough to $q_{0}$. In this paper, we want to answer to this question.

Let us recall several notations which are necessary for later use. For every distribution $r$ on $S, r G_{T}^{m}$ on $S$ is given by

$$
\left(r G_{T}{ }^{m}\right)(x):=\sum_{y \in S} r(y) G_{T}^{m}(y, x)
$$

where $G_{T}^{m}$ is the $m$-th power of the Gibbs matrix $G_{T}$ with temperature $T$, that is,

$$
G_{T}^{m}(x, y):=\sum_{z_{1}, \cdots, z_{m} \in S} G_{T}\left(x, z_{1}\right) \cdots G_{T}\left(z_{m}, y\right)
$$

The Gibbs matrix $G_{T}=\left(G_{T}(x, y)\right)_{x, y \in S}$ is given by definition as

2000 Mathematics Subject Classification. Primary 60J05; Secondary 62L20.

Supported by the Grant-in-Aid for the Scientific Research, (A)19204004,(C)21540207, Japan Society for the Promotion of Science.

* Corresponding author. E-mail: urakawa@math.is.tohoku.ac.jp 


$$
G_{T}(x, y)= \begin{cases}P(x, y) A_{T}(x, y) & \text { if } x \neq y \\ 1-\sum_{z \neq x} P(x, z) A_{T}(x, z) & \text { if } x=y\end{cases}
$$

$P=(P(x, y))_{x, y \in S}$ is the generating probability matrix and $A_{T}=\left(A_{T}(x, y)\right)_{x, y \in S}$ is the accepting matrix, which is given by

$$
A_{T}(x, y)=g\left(\frac{q_{T}(y)}{q_{T}(x)}\right)
$$

where $g(u)$ is the accepting function on the open interval $(0, \infty)$ into the interval $(0,1]$ satisfying that

$$
g(u)=u g\left(\frac{1}{u}\right), \quad 0<u<\infty .
$$

Furthermore, let us recall the definition of Gibbs distribution $q_{T}$ :

$$
q_{T}(x)=\frac{e^{-\frac{1}{T} F(x)}}{Z}, \quad x \in S,
$$

where $Z$ is the Boltzman constant which is by definition

$$
Z=\sum_{y \in S} e^{-\frac{1}{T} F(y)} .
$$

Then, $G_{T}$ is reversible with respect to $q_{T}$, that is,

$$
q_{T}(x) G_{T}(x, y)=q_{T}(y) G_{T}(y, x), \quad \forall \quad x, y \in S,
$$

and $q_{T}$ is the equilibrium distribution, that is,

$$
\sum_{x \in S} q_{T}(x) G_{T}(x, y)=q_{T}(y), \quad \forall \quad y \in S .
$$

It is well known that $G_{T}$ is irreducible, that is, for all $x$ and $y$ in $S$, there exists a positive integer $k$ such that $G_{T}{ }^{k}(x, y)>0$. Furthermore, $G_{T}$ is aperiodic, that is, $\operatorname{gcd}\left\{k ; G_{T}{ }^{k}(x, y)>0\right\}=1$. Due to ergodic theorem, it holds (cf. [1]) that

Theorem 1.1. For all initial distribution $r$,

$$
\lim _{m \rightarrow \infty} r G_{T}{ }^{m}=q_{T}
$$

Now, let us recall the notion of the total variation distance between two probability distributions $\mu$ and $\mu^{\prime}$ on $S$ :

$$
\begin{aligned}
\left\|\mu-\mu^{\prime}\right\|_{T V} & :=\sup \left\{\left|\mu(A)-\mu^{\prime}(A)\right|: A \in \mathscr{B}\right\} \\
& =\frac{1}{2} \sum_{x \in S}\left|\mu(x)-\mu^{\prime}(x)\right|,
\end{aligned}
$$

where $\mathcal{B}$ is the $\sigma$-algebra of all Borel sets in $S$. Diaconis and Stroock [3] estimated the total variation distance between any reversible Markov chain $G$ (with respect to $\pi$ ) and the stationary distribution $\pi$ in terms of the eigenvalues of $G$ (See the next section). Applying their result to the Boltzmann machine, we have:

Theorem 1.2. For every $T>0$ and $m=1,2, \ldots$,

$$
\left\|r G_{T}{ }^{m}-q_{0}\right\|_{T V} \leq \frac{\sqrt{|S|}}{2} \beta_{*}{ }^{m}\left(\left|S_{0}\right|+\sum_{y \in S \backslash S_{0}} e^{\frac{1}{2 T}\left(F(y)-F_{\min }\right)}\right)+\frac{\sum_{x \in S \backslash S_{0}} e^{-\frac{1}{T}\left(F(x)-F_{\min }\right)}}{\left|S_{0}\right|+\sum_{x \in S \backslash S_{0}} e^{-\frac{1}{T}\left(F(x)-F_{\min }\right)}},
$$

where $0 \leq \beta_{*}:=\max \left\{\beta_{1},\left|\beta_{a-1}\right|\right\}<1$. Here, we write the eigenvalues of the Gibbs matrix $G_{T}$ as

$$
1=\beta_{0}>\beta_{1} \geq \cdots \geq \beta_{a-1} \geq-1, \quad(\text { where } a:=|S|) \text {. }
$$

Applying this estimation (1.9) to the simplest set $S$ of states with $|S|=4$, we can concretely estimate $T$ and $m$ such that

$$
\left\|r G_{T}{ }^{m}-q_{0}\right\|_{T V}<\epsilon=0.1 \text {. }
$$

For the case $|S|=8$, see Section Four. In the case $|S|=4$, more precisely, let us consider the function $F$ on $S=\{a, b, c, d\}$ with four values, say

$$
0>-v_{1}>-v_{2}>-w_{12}-v_{1}-v_{2}
$$

without loss of generality. We choose the accepting function $g$ as 


$$
g(u)=\frac{u}{1+u}=\frac{1}{1+u^{-1}}=: \tau(u) .
$$

How much $0<T<\infty$ and $m$ can one take to search the point of $S=\{a, b, c, d\}$ at which $F$ attains the minimum value $-w_{12}-v_{1}-v_{2}$ by simulated annealing? Our answer is this:

Theorem 1.3. If $0<T \leq \frac{w_{12}+v_{1}}{2.70806}$ and

$$
m \geq 31.2415 \times \frac{w_{12}+v_{1}+v_{2}}{T}+169.207
$$

in the case $w_{12}>0$, or

$$
m \geq 1.06382 \times \frac{w_{12}+v_{1}+v_{2}}{T}+5.76176
$$

in the case $w_{12}<0$, then

$$
\left\|r G_{T}{ }^{m}-q_{0}\right\|_{T V}<\frac{1}{10}=0.1 .
$$

Remark 1.4. By Theorem 1.3, if we take $T$ and $m$ as in this theorem, the Boltzmann machine can search the point of $S$ at which $F$ attains the minimum with probability 90\%. This estimation explains the character of the Boltzmann machine very well. Indeed, in order to estimate the convergence rates of the simulated annealing lower that 0.1 , one should set the temperature $T$ lower than $\frac{w_{12}+v_{1}}{2.70806}$. But, if one sets for the temperature $T$ to be too low, a large transition time $m$ is necessary.

Remark 1.5. The right hand side of (1.9) in Theorem 1.2 does not depend on the initial state $r$ on S. The first term of the right hand side converges to zero if $m$ tends to infinity since $0 \leq \beta_{*}<1$. The second term converges to zero if $T$ tends to zero. Be careful that the factor of the first term, $\sum_{y \in S \backslash S_{0}} e^{\frac{1}{2 T}\left(F(y)-F_{\min }\right)}$ goes to infinity if $T$ tends to zero.

\section{Proof of Theorem 1.2}

We first prove

Theorem 2.1.

$$
\begin{aligned}
\left\|q_{T}-q_{0}\right\|_{T V} & =\frac{\left|S_{0}\right|}{Z} e^{-\frac{1}{T} F_{\min }}-1=\frac{1}{Z} \sum_{x \in S \backslash S_{0}} e^{-\frac{1}{T} F(x)} \\
& =\frac{\sum_{x \in S \backslash S_{0}} e^{-\frac{1}{T}\left(F(x)-F_{\min }\right)}}{\left|S_{0}\right|+\sum_{x \in S \backslash S_{0}} e^{-\frac{1}{T}\left(F(x)-F_{\min }\right)}}
\end{aligned}
$$

Proof. By (1.8), we have

$$
\left\|q_{T}-q_{0}\right\|_{T V}=\frac{1}{2} \sum_{x \in S}\left|\frac{1}{Z} e^{-\frac{1}{T} F(x)}-\frac{1}{\left|S_{0}\right|} \chi_{S_{0}}(x)\right|,
$$

where $\chi_{S_{0}}$ is the characteristic function of $S_{0}$ in $S$. The right hand side of (2.3) coincides with

$$
\frac{1}{2} \sum_{x \in S_{0}}\left|\frac{1}{Z} e^{-\frac{1}{T} F(x)}-\frac{1}{\left|S_{0}\right|} \chi_{S_{0}}(x)\right|+\frac{1}{2} \sum_{x \in S \backslash S_{0}}\left|\frac{1}{Z} e^{-\frac{1}{T} F(x)}-\frac{1}{\left|S_{0}\right|} \chi_{S_{0}}(x)\right| \text {, }
$$

which is equal to

$$
\frac{\left|S_{0}\right|}{2 Z}\left|e^{-\frac{1}{T} F_{\min }}-\frac{Z}{\left|S_{0}\right|}\right|+\frac{1}{2 Z} \sum_{x \in S \backslash S_{0}} e^{-\frac{1}{T} F(x)}
$$

But, we have

$$
\begin{aligned}
Z & =\sum_{x \in S} e^{-\frac{1}{T} F(x)}=\sum_{x \in S_{0}} e^{-\frac{1}{T} F(x)}+\sum_{x \in S \backslash S_{0}} e^{-\frac{1}{T} F(x)} \\
& =\left|S_{0}\right| e^{-\frac{1}{T} F_{\min }}+\sum_{x \in S \backslash S_{0}} e^{-\frac{1}{T} F(x)}
\end{aligned}
$$

which implies that

$$
-e^{-\frac{1}{T} F_{\min }}+\frac{Z}{\left|S_{0}\right|}=\frac{1}{\left|S_{0}\right|} \sum_{x \in S \backslash S_{0}} e^{-\frac{1}{T} F(x)} \geq 0,
$$


so that we have (2.1). By (2.5),

$$
\frac{1}{Z} \sum_{x \in S \backslash S_{0}} e^{-\frac{1}{T} F(x)}=\frac{\sum_{x \in S \backslash S_{0}} e^{-\frac{1}{T} F(x)}}{\left|S_{0}\right| e^{-\frac{1}{T} F_{\min }}+\sum_{x \in S \backslash S_{0}} e^{-\frac{1}{T} F(x)}}=\frac{\sum_{x \in S \backslash S_{0}} e^{-\frac{1}{T}\left(F(x)-F_{\min }\right)}}{\left|S_{0}\right|+\sum_{x \in S \backslash S_{0}} e^{-\frac{1}{T}\left(F(x)-F_{\min }\right)}},
$$

which implies (2.2).

Next, we have

\section{Theorem 2.2.}

$$
\left\|r G_{T}{ }^{m}-q_{T}\right\|_{T V} \leq \beta_{*}{ }^{m} \cdot \frac{1}{2} \sum_{y \in S} r(y)\left(\frac{1-q_{T}(y)}{q_{T}(y)}\right)^{\frac{1}{2}} .
$$

Before going into the proof of Theorem 2.2, let us recall the results due to Diaconis and Stroock (cf. [3]) of the transition probability $G(x, y)$ for an irreducible Markov chain reversible relative to the stationary distribution $\pi$, that is

$$
\pi(x) G(x, y)=\pi(y) G(y, x), \quad \forall x, y \in S .
$$

We denote all the eigenvalues of the matrix $G=(G(x, y))_{x, y \in S}$ by

$$
1=\beta_{0}>\beta_{1} \geq \beta_{2} \geq \cdots \geq \beta_{a-1} \geq-1,
$$

where $a=|S|$. Then, it holds (cf. [3]) that

$$
2\left\|G^{m}(x, \cdot)-\pi(\cdot)\right\|_{T V} \leq\left(\frac{1-\pi(x)}{\pi(x)}\right)^{1 / 2} \beta_{*}{ }^{m},
$$

where $\beta_{*}:=\max \left\{\beta_{1},\left|\beta_{a-1}\right|\right\}$.

By applying (2.9) to $G_{T}$, we have

$$
2\left\|G_{T}{ }^{m}(x, \cdot)-q_{T}(\cdot)\right\|_{T V} \leq\left(\frac{1-q_{T}(x)}{q_{T}(x)}\right)^{1 / 2} \beta_{*}{ }^{m} .
$$

(Proof of Theorem 2.2). We have by definition and (1.8)

$$
\begin{aligned}
\left\|r G_{T}{ }^{m}-q_{T}\right\|_{T V} & =\left\|\sum_{y \in S} r(y) G_{T}{ }^{m}(y, \cdot)-q_{T}(\cdot)\right\|_{T V} \\
& =\frac{1}{2} \sum_{x \in S}\left|\sum_{y \in S} r(y) G_{T}{ }^{m}(y, x)-q_{T}(x)\right| \\
& =\frac{1}{2} \sum_{x \in S}\left|\sum_{y \in S} r(y)\left(G_{T}{ }^{m}(y, x)-q_{T}(x)\right)\right| \\
& \leq \frac{1}{2} \sum_{x \in S} \sum_{y \in S} r(y)\left|G_{T}{ }^{m}(y, x)-q_{T}(x)\right| \\
& =\sum_{y \in S} r(y) \frac{1}{2} \sum_{x \in S}\left|G_{T}{ }^{m}(y, x)-q_{T}(x)\right| \\
& =\sum_{y \in S} r(y)\left\|G_{T}{ }^{m}(y, \cdot)-q_{T}(\cdot)\right\|_{T V} \\
& \leq \sum_{y \in S} r(y) \frac{1}{2}\left(\frac{1-q_{T}(y)}{q_{T}(y)}\right)^{1 / 2} \beta_{*}{ }^{m},
\end{aligned}
$$

which follows from (2.10). (2.11) is the desired one.

To estimate the right hand side of (2.8), we have

Proposition 2.3. (1) For the upper bound,

$$
\sum_{y \in S} r(y)\left(\frac{1-q_{T}(y)}{q_{T}(y)}\right)^{1 / 2} \leq|S|^{1 / 2}\left(\left|S_{0}\right|+\sum_{y \in S \backslash S_{0}} e^{\frac{1}{2 T}\left(F(y)-F_{\min }\right)}\right) .
$$

(2) For the lower bound, 


$$
\sum_{y \in S} r(y)\left(\frac{1-q_{T}(y)}{q_{T}(y)}\right)^{1 / 2} \geq\left|S_{0}\right|^{1 / 2} \sum_{y \in S \mid S_{0}} r(y) e^{\frac{1}{2 T}\left(F(y)-F_{\min }\right)} .
$$

Proof. (1) Since $0 \leq r(y) \leq 1$ and $q_{T}(y) \leq 1$, we have

$$
\sum_{y \in S} r(y)\left(\frac{1-q_{T}(y)}{q_{T}(y)}\right)^{1 / 2} \leq \sum_{y \in S} q_{T}(y)^{-1 / 2}=Z^{1 / 2} \cdot \sum_{y \in S} e^{\frac{1}{2 T} F(y)} .
$$

Here, by definition of $Z$, we have

$$
e^{\frac{1}{T} F_{\min }} Z=\left|S_{0}\right|+\sum_{z \in S \backslash S_{0}} e^{-\frac{1}{T}\left(F(z)-F_{\min }\right)} \leq\left|S_{0}\right|+\left|S \backslash S_{0}\right|=|S| .
$$

Then, by (2.15), we have

$$
\begin{aligned}
Z^{1 / 2} \cdot \sum_{y \in S} e^{\frac{1}{2 T} F(y)} & \leq|S|^{1 / 2} e^{-\frac{1}{2 T} F_{\min }} \sum_{y \in S} e^{\frac{1}{2 T} F(y)} \\
& =|S|^{1 / 2} \sum_{y \in S} e^{\frac{1}{2 T}\left(F(y)-F_{\min }\right)} \\
& =|S|^{1 / 2}\left(\left|S_{0}\right|+\sum_{y \in S \backslash S_{0}} e^{\frac{1}{2 T}\left(F(y)-F_{\min }\right)}\right),
\end{aligned}
$$

which is (2.12).

For (2), by definition of $q_{T}$, we have, for every $y_{0} \in S \backslash S_{0}$,

$$
\frac{1-q_{T}\left(y_{0}\right)}{q_{T}\left(y_{0}\right)}=\frac{1-e^{-\frac{1}{T} F\left(y_{0}\right)} / Z}{e^{-\frac{1}{T} F\left(y_{0}\right)} / Z}=e^{\frac{1}{T} F\left(y_{0}\right)} Z-1 .
$$

Here, we have

$$
\begin{aligned}
e^{\frac{1}{T} F\left(y_{0}\right)} Z & =e^{\frac{1}{T} F\left(y_{0}\right)}\left(\left|S_{0}\right| e^{-\frac{1}{T} F_{\min }}+\sum_{y \in S \backslash S_{0}} e^{-\frac{1}{T} F(y)}\right) \\
& =\left|S_{0}\right| e^{\frac{1}{T}\left(F\left(y_{0}\right)-F_{\min }\right)}+\sum_{y \in S \backslash S_{0}} e^{\frac{1}{T}\left(F\left(y_{0}\right)-F(y)\right)} \\
& =\left|S_{0}\right| e^{\frac{1}{T}\left(F\left(y_{0}\right)-F_{\min }\right)}+1+\sum_{y_{0} \neq y \in S \backslash S_{0}} e^{\frac{1}{T}\left(F\left(y_{0}\right)-F(y)\right)} \\
& \geq\left|S_{0}\right| e^{\frac{1}{T}\left(F\left(y_{0}\right)-F_{\min }\right)}+1 .
\end{aligned}
$$

Thus, we have, for every $y_{0} \in S \backslash S_{0}$,

$$
\frac{1-q_{T}\left(y_{0}\right)}{q_{T}\left(y_{0}\right)} \geq\left|S_{0}\right| e^{\frac{1}{T}\left(F\left(y_{0}\right)-F_{\min }\right)},
$$

which yields (2.13).

By Theorems 2.1, 2.2 and Proposition 2.3, we have Theorem 1.2.

\section{The Bolzmann Machine of Neural Networks}

Now we apply our estimate to the Boltzmann machine coming from neural networks. The set $S$ of states is given by $S=\left\{\left(x_{1}, x_{2}, \cdots, x_{n}\right) ; x_{i}=0\right.$ or $\left.1(i=1, \cdots, n)\right\}\left(|S|=2^{n}\right)$, and our function $F$ on $S$ is given by

$$
F(x)=-\frac{1}{2} \sum_{i, j=1}^{n} w_{i j} x_{i} x_{j}-\sum_{i=1}^{n} v_{i} x_{i}
$$

where $w_{i i}=0$ and $w_{i j}=w_{j i}(i, j=1, \ldots, n)$. For two states $x$ and $y$ in $S$, the transition probability from $x$ to $y$ is given by

$$
P(x, y)= \begin{cases}\frac{1}{n} & \text { if } x \sim y \\ 0 & \text { otherwise }\end{cases}
$$


where $x \sim y$ stands that the Hamming distance on $S$ between $x$ and $y$ is 1 . The accepting matrix $A_{T}$ is given by

$$
A_{T}(x, y)=\tau\left(-\frac{F(y)-F(x)}{T}\right)
$$

where the accepting function $\tau$ is given by $(1.12)$. For $x=\left(x_{1}, x_{2}, \ldots, x_{n}\right) \quad$ and $y=x(k)=$ $\left(x_{1}, x_{2}, \ldots, x_{k-1}, 1-x_{k}, x_{k+1}, \ldots, x_{n}\right)$, we have

$$
A_{T}(x, x(k))=\tau\left(-\frac{\left(2 x_{k}-1\right) U_{k}(x)}{T}\right)
$$

where

$$
u_{k}=U_{k}(x)=\sum_{j=1}^{n} w_{k j} x_{j}+v_{k} \quad(k=1, \ldots, n)
$$

which is called the interior voltage of $k$-th neuron. All the elements belonging to the set $S$ are ordered as small as the function $N(x)=\sum_{k=1}^{n} k x_{k}\left(x=\left(x_{1}, \ldots, x_{n}\right) \in S\right)$.

In the sequel of this section, we treat with the case $|S|=4(n=2)$, and will treat with the case $|S|=8(n=3)$ in the next section. In the case $|S|=4(n=2)$, we can calculate $P, A_{T}$ and $G_{T}$ as follows.

$$
P=\left(\begin{array}{cccc}
0 & \frac{1}{2} & \frac{1}{2} & 0 \\
\frac{1}{2} & 0 & 0 & \frac{1}{2} \\
\frac{1}{2} & 0 & 0 & \frac{1}{2} \\
0 & \frac{1}{2} & \frac{1}{2} & 0
\end{array}\right)
$$

and

$$
G_{T}=\left(\begin{array}{cccc}
1-\frac{1}{2} A-\frac{1}{2} B & \frac{1}{2} A & \frac{1}{2} B & 0 \\
\frac{1}{2}-\frac{1}{2} A & \frac{1}{2}+\frac{1}{2} A-\frac{1}{2} D & 0 & \frac{1}{2} D \\
\frac{1}{2}-\frac{1}{2} B & 0 & \frac{1}{2}+\frac{1}{2} B-\frac{1}{2} C & \frac{1}{2} C \\
0 & \frac{1}{2}-\frac{1}{2} D & \frac{1}{2}-\frac{1}{2} C & \frac{1}{2} C+\frac{1}{2} D
\end{array}\right)
$$

where

$$
A=\tau\left(\frac{v_{1}}{T}\right), \quad B=\tau\left(\frac{v_{2}}{T}\right), \quad C=\tau\left(\frac{w_{12}+v_{1}}{T}\right), \quad D=\tau\left(\frac{w_{12}+v_{2}}{T}\right) .
$$

By using "Mathematica", one can compute the eigenvalues of $G_{T}$ as follows:

$$
\left\{1>\frac{1}{2}(1+\sqrt{(A-C)(B-D)})>\frac{1}{2}(1-\sqrt{(A-C)(B-D)})>0\right\} .
$$

Thus, we obtain

$$
\beta_{*}=\frac{1}{2}(1+\sqrt{(A-C)(B-D)}) .
$$

Now, we take $\epsilon=\frac{1}{10},|S|=4,\left|S_{0}\right|=1$ and $\left|S \backslash S_{0}\right|=3$. By Theorems 1.2 or 2.1, if $0<T \leq T_{0}$,

$$
\left\|q_{T}-q_{0}\right\|_{T V} \leq \frac{1}{20}
$$

where

$$
\begin{aligned}
T_{0} & =\frac{F_{1}-F_{\min }}{-\log \epsilon+\log 2-\log |S|+\log \left|S \backslash S_{0}\right|} \\
& =\frac{w_{12}+v_{1}}{2.70806} .
\end{aligned}
$$

Here, we put

$$
F_{\max }=0>F_{2}=-v_{1}>F_{1}=-v_{2}>F_{\min }=-w_{12}-v_{1}-v_{2}
$$

satisfying that

$$
0<v_{1}<v_{2} \text {, and } 0<w_{12}+v_{1} \text {. }
$$


On the other hand, for the above $T>0$, by Theorems 1.1 or 2.2, and Proposition 2.3, to satisfy $\left\|r G_{T}{ }^{m}-q_{T}\right\|_{T V}<\frac{\epsilon}{2}$, it suffices to take for any $m$ satisfying that

$$
\beta_{*}{ }^{m} \frac{|S|^{3 / 2}}{2} e^{\frac{1}{2 T}\left(F_{\max }-F_{\min }\right)}<\frac{\epsilon}{2}
$$

which is equivalent to the following:

$$
\begin{aligned}
m & >\frac{1}{-\log \beta_{*}}\left\{-\log \epsilon+\frac{3}{2} \log |S|+\frac{1}{2 T}\left(F_{\max }-F_{\min }\right)\right\} \\
& =\frac{1}{-\log \beta_{*}}\left\{\log 10+3 \log 2+\frac{1}{2 T}\left(w_{12}+v_{1}+v_{2}\right)\right\} \\
& =\frac{1}{-\log \beta_{*}}\left\{4.38203+\frac{1}{2 T}\left(w_{12}+v_{1}+v_{2}\right)\right\},
\end{aligned}
$$

since $\epsilon=\frac{1}{10},|S|=4, F_{\max }=0$, and $F_{\min }=-w_{12}-v_{1}-v_{2}$.

Here, $\beta_{*}=\frac{1}{2}(1+x)$ with $0<x<1$ which is given by

$$
\begin{aligned}
x= & \sqrt{\left(\tau\left(\frac{v_{1}}{T_{0}}\right)-\tau\left(\frac{w_{12}+v_{1}}{T_{0}}\right)\right)\left(\tau\left(\frac{v_{2}}{T_{0}}\right)-\tau\left(\frac{w_{12}+v_{2}}{T_{0}}\right)\right)} \\
= & \left\{\frac{e^{-\frac{v_{1}}{T_{0}}}}{1+e^{-\frac{v_{1}}{T_{0}}}}\right\}^{1 / 2}\left\{\frac{e^{-\frac{v_{2}}{T_{0}}}}{1+e^{-\frac{v_{2}}{T_{0}}}}\right\}^{1 / 2} \\
& \times\left\{\frac{1}{1+e^{-\frac{w_{12}+v_{1}}{T_{0}}}}\right\}^{1 / 2}\left\{\frac{1}{1+e^{-\frac{w_{12}+v_{2}}{T_{0}}}}\right\}^{1 / 2}\left|e^{-\frac{w_{12}}{T_{0}}}-1\right| \\
= & \left.\left\{\frac{e^{\frac{v_{1}}{T_{0}}}}{1+e^{\frac{v_{1}}{T_{0}}}}\right\}^{1 / 2}\left\{\frac{e^{\frac{v_{2}}{T_{0}}}}{1+e^{\frac{v_{2}}{T_{0}}}}\right\}^{1 / 2}\right|^{1 / 2}\left\{\frac{1}{1+2}\left|e^{\frac{w_{12}}{T_{0}}}-1\right| .\right. \\
& \left.\times\left\{\frac{1}{1+e^{\frac{w_{12}+v_{1}}{T_{0}}}}\right\}^{\frac{w_{0}}{T_{0}}}\right\}^{1 / 2}\{
\end{aligned}
$$

If $w_{12} \geq 0$, by (3.13), we have

$$
\frac{1}{-\log \beta_{*}} \leq 62.9842 .
$$

And if $w_{12}<0$, by (3.14), we have

$$
\frac{1}{-\log \beta_{*}} \leq 2.12764
$$

We obtain the desired lower bound of $m$.

\section{The Boltzmann Machine for the Case $|S|=8$}

In this section, we retain the general setting for the Boltzmann machine for neural networks and set $|S|=8(n=3)$.

For simplicity, we assume that

$$
v_{1}=v_{2}=v_{3}=v>0, \quad w_{12}=w_{13}=w_{23}=w>0 .
$$

Then, the matrices $P, A_{T}$, and $G_{T}$ can be calculated as follows.

$$
P=\left(\begin{array}{cccccccc}
0 & \frac{1}{3} & \frac{1}{3} & 0 & \frac{1}{3} & 0 & 0 & 0 \\
\frac{1}{3} & 0 & 0 & \frac{1}{3} & 0 & \frac{1}{3} & 0 & 0 \\
\frac{1}{3} & 0 & 0 & \frac{1}{3} & 0 & 0 & \frac{1}{3} & 0 \\
0 & \frac{1}{3} & \frac{1}{3} & 0 & 0 & 0 & 0 & \frac{1}{3} \\
\frac{1}{3} & 0 & 0 & 0 & 0 & \frac{1}{3} & \frac{1}{3} & 0 \\
0 & \frac{1}{3} & 0 & 0 & \frac{1}{3} & 0 & 0 & \frac{1}{3} \\
0 & 0 & \frac{1}{3} & 0 & \frac{1}{3} & 0 & 0 & \frac{1}{3} \\
0 & 0 & 0 & \frac{1}{3} & 0 & \frac{1}{3} & \frac{1}{3} & 0
\end{array}\right),
$$




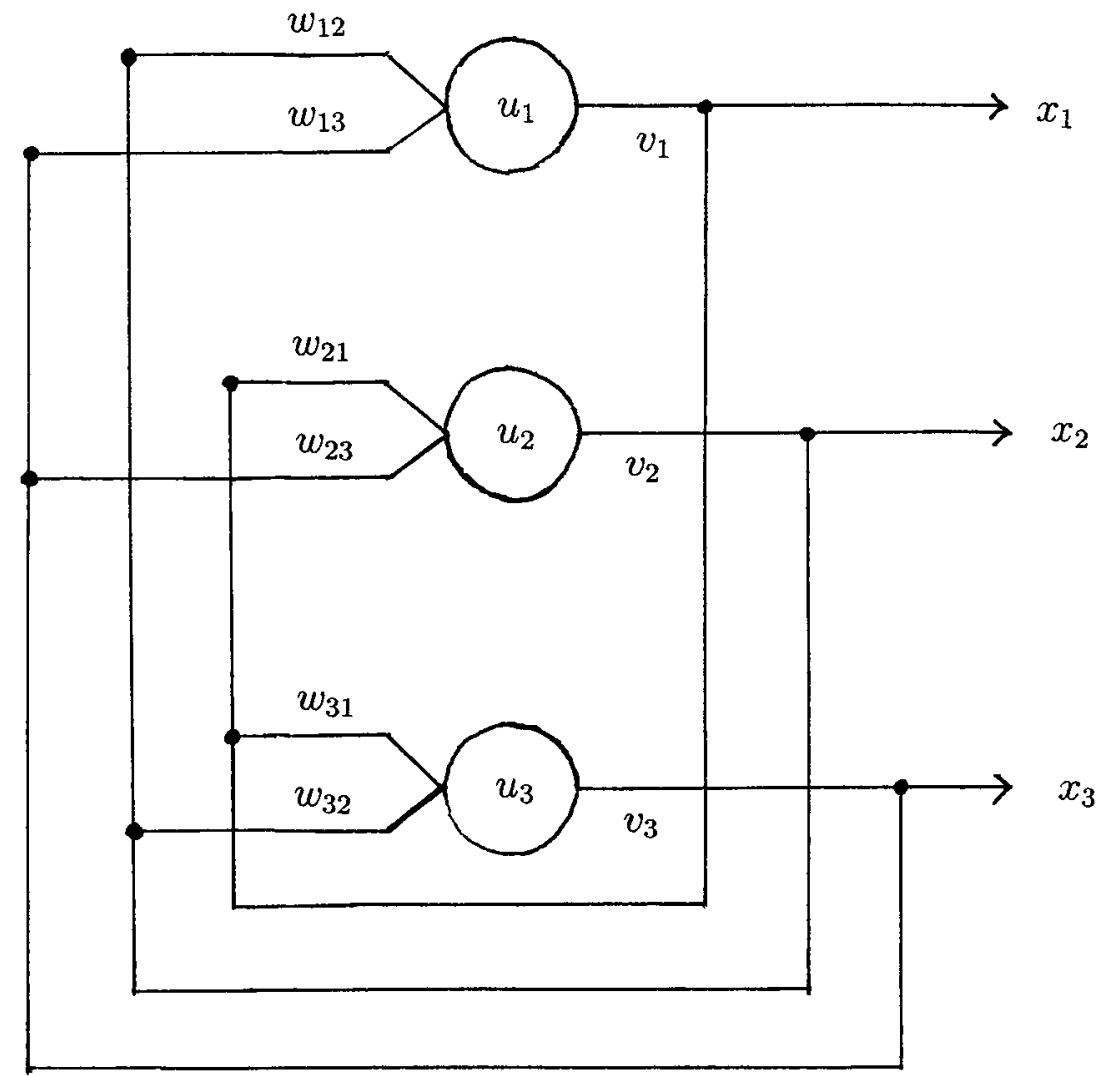

Fig. 1. Neural Network for $n=3$.

and $G_{T}$ is equal to

$$
\left(\begin{array}{cccccccc}
1-3 a & a & a & 0 & a & 0 & 0 & 0 \\
\frac{1}{3}-a & \frac{2}{3}+a-2 b & 0 & b & 0 & b & 0 & 0 \\
\frac{1}{3}-a & 0 & \frac{2}{3}+a-2 b & b & 0 & 0 & b & 0 \\
0 & \frac{1}{3}-b & \frac{1}{3}-b & \frac{1}{3}+2 b-c & 0 & 0 & 0 & c \\
\frac{1}{3}-a & 0 & 0 & 0 & \frac{2}{3}+a-2 b & b & b & 0 \\
0 & \frac{1}{3}-b & 0 & 0 & \frac{1}{3}-b & \frac{1}{3}+2 b-c & 0 & c \\
0 & 0 & \frac{1}{3}-b & 0 & \frac{1}{3}-b & 0 & \frac{1}{3}+2 b-c & c \\
0 & 0 & 0 & \frac{1}{3}-c & 0 & \frac{1}{3}-c & \frac{1}{3}-c & 3 c
\end{array}\right),
$$

where $a=\frac{1}{3} \tau\left(\frac{v}{T}\right), b=\frac{1}{3} \tau\left(\frac{w+v}{T}\right)$, and $c=\frac{1}{3} \tau\left(\frac{2 w+v}{T}\right)$.

By using "Mathematica", the eigenvalues of $G_{T}$ are given as follows:

$$
\left.\left\{1,0, \frac{1}{2}(1+a-c \pm \alpha) \text { (with multiplicities } 2\right), \frac{1}{2}(1-a+2 c \pm \beta)\right\},
$$

where

$$
\begin{aligned}
& \alpha=\frac{1}{3} \sqrt{1+6 a+9 a^{2}-12 b-72 a b+108 b^{2}+6 c+18 a c-72 b c+9 c^{2}} \\
& \beta=\frac{1}{3} \sqrt{1-12 a+36 a^{2}+24 b-72 a b-12 c+72 a c-72 b c+36 c^{2}} .
\end{aligned}
$$

Then, we obtain that the $\beta^{*}$ in Theorem 1.2 is given by

$$
\beta^{*}=\max \left\{\frac{1}{2}(1+a-c+\alpha), \frac{1}{2}(1-a+2 c+\beta)\right\} .
$$

Note that $0<\beta^{*}<1$.

The set of values of the function $F$ is given as 


$$
F_{\max }=0>F_{2}=-v>F_{1}=-w-2 v>F_{\min }=-3 w-3 v,
$$

and $S_{0}=\left\{x \in S ; F(x)=F_{\min }\right\}=\{(1,1,1)\}$, so we have $\left|S_{0}\right|=1$, and $\left|S \backslash S_{0}\right|=7$.

Then, the right hand side of (1.9) of Theorem 1.2 can be calculated as follows: The first term of the right hand side of (1.9) coincides with

$$
\sqrt{2} \beta^{* m}\left(1+e^{\frac{1}{2 T}(3 w+3 v)}+3 e^{\frac{1}{2 T}(3 w+2 v)}+3 e^{\frac{1}{2 T}(2 w+v)}\right) \leq 8 \sqrt{2} \beta^{* m} e^{\frac{1}{2 T}(3 w+3 v)},
$$

and the second term of the right hand side of (1.9) coincides with

$$
\frac{e^{-\frac{1}{T}(3 w+3 v)}+3 e^{-\frac{1}{T}(3 w+2 v)}+3 e^{-\frac{1}{T}(2 w+v)}}{1+e^{-\frac{1}{T}(3 w+3 v)}+3 e^{-\frac{1}{T}(3 w+2 v)}+3 e^{-\frac{1}{T}(2 w+v)}} \leq 7 e^{-\frac{1}{T}(2 w+v)} .
$$

Therefore, the right hand side of (1.9) is smaller than or equal to

$$
8 \sqrt{2} \beta^{* m} e^{\frac{1}{2 T}(3 w+2 v)}+7 e^{-\frac{1}{T}(2 w+v)} .
$$

Thus, we obtain

Proposition 4.1. For every $T>0$ and $m=1,2, \ldots$,

$$
\left\|r G_{T}^{m}-q_{0}\right\|_{T V} \leq 8 \sqrt{2} \beta^{* m} e^{\frac{1}{2 T}(3 w+2 v)}+7 e^{-\frac{1}{T}(2 w+v)} .
$$

Here, $\beta^{*}$ is given by (4.2).

\section{REFERENCES}

[1] Aarts, E., and Korst, J., Simulated Annealing and Boltzmann Machine, A Stochastic Approach to Combinatorial Optimization and Neural Computing, Wiley Series in Discrete Math. and Optimization, Wiley, New York, (1989).

[2] Catoni, O., Rates of convergence for sequential annealing: a large deviation approach, In: Simulated annealing, Wiley Series in Discrete Math. and Optimization, Wiley, New York, (1992), 25-35.

[3] Diaconis, P., and Stroock, D., "Geometric bounds for eigenvaluesof Markov chains," Ann. Appl. Probability, 1: 36-61 (1991).

[4] Douc, R., Moulines, E., and Rosenthal, J. S., "Quantitative bounds on convergence of time-inhomogeneous Markov chains," Ann. Applied Probability, 14: 1643-1665 (2004).

[5] Fill, J. A., "Eigenvalue bounds on convergence to stationarity for nonreversible Markov chains, with an application to the exclusion process," Ann. Appl. Probability, 1: 62-87 (1991).

[6] Jerrum, M., Counting, Sampling and Integrating: Algorithms and Complexity, Lectures in Math., ETH Zürich, Birkäuser, 2003.

[7] Jones, G. L., and Hobert, J. P., "Honest exploration of intractable probability distributions via Markov chain Monte Carlo," Statistic Science, 16: 312-334 (2001).

[8] Kirkpatrick, S., Gelatt, C. D., and Vecchi, M. P., “Optimization by simulated annealing,” Science, 220: 671-680 (1983).

[9] Kirkpatrick, S., "Optimization by simulated annealing: quantitative studies," J. Statist. Physics, 34: $975-986$ (1984).

[10] Márquez, D., "Convergence rates for annealing diffusion processes," Ann. Applied Probability, 7: 1118-1139 (1997).

[11] Meyn, S. P., and Tweedie, R. L., "Computable bounds for geometric convergence rates of Markov chains," Ann. Applied Probability, 4: 981-1011 (1994).

[12] Mengersen, K. L., and Tweedie, R. L., "Rates of convergence of the Hastings and Metropolis algorithms," Ann. Statistics, 24: 101-121 (1996).

[13] Roberts, G. O., and Tweedie, R. L., "Bounds on regeneration times and convergence rates for Markov chains," Stochastic Process. Appl., 80: 211-229 (1999); Corrigendum, 91: 337-338 (2001).

[14] Rosenthal, J. S., "Minorization conditions and convergence rates for Markov chain Monte Carlo," J. Amer. Statist. Assoc., 90: 558-566 (1995).

[15] — "Quantitative convergence rates of Markov chains: a simple account," Electron. Comm. Probab., 7: 123-128 (2002).

[16] Saloff-Coste, L., Lectures on Finite Markov Chains, Lectures on Probability Theory and Statistics, Springer-Verlag, Berlin Heidelberg (1997).

[17] — - "Probability on groups: random walks and invariant diffusions," Notices Amer. Math. Soc., 48: $968-977$ (2001).

[18] - Random Walks on Finite Groups, Encyclopaedia Math. Sci., Springer-Verlag, Berlin Heidelberg, (2004), $263-346$.

[19] Sinclair, A., and Jerrum, M., "Approximate counting, uniform generation and rapidly mixing Markov chains," Information Computation, 82: 93-133 (1989).

[20] Urakawa, H., "Convergence rates to equilibrium of the heat kernels on compact Riemannian manifolds," Indiana Univ. Math. J., 55: 259-288 (2006). 